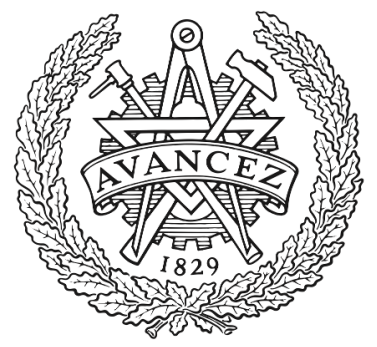

CHALMERS

UNIVERSITY OF TECHNOLOGY

\title{
Mm-Wave contactless connection for MMIC integration in gap waveguides
}

Downloaded from: https://research.chalmers.se, 2023-04-26 12:21 UTC

Citation for the original published paper (version of record):

Aljarosha, A., Maaskant, R., Uz Zaman, A. et al (2016). Mm-Wave contactless connection for MMIC integration in gap waveguides. 2016 IEEE Antennas and Propagation Society International Symposium, APSURSI 2016, Fajardo, Puerto Rico, 26 June - 1 July 2016: 253-254.

http://dx.doi.org/10.1109/APS.2016.7695835

N.B. When citing this work, cite the original published paper. 


\section{mm-Wave Contactless Connection for MMIC Integration in Gap Waveguides}

\author{
Alhassan Aljarosha \\ Department of Signals and Systems \\ Chalmers University of Technology \\ Gothenburg, Sweden \\ alhalj@ student.chalmers.se
}

\author{
Rob Maaskant, Ashraf Uz Zaman and Per-Simon Kildal \\ Department of Signals and Systems \\ Chalmers University of Technology \\ Gothenburg, Sweden \\ rob.maaskant@chalmers.se,zaman@chalmers.se,per-simon.kildal@chalmers.se
}

\begin{abstract}
A contactless, broadband and low-loss microstripto-groove gap waveguide transition operating at W-band is presented. The principle of operation is based on transforming EM fields from the SIW to the ridge gap waveguide mode via electromagnetic coupling. This is advantageous, since the proposed solution avoids the use of metal contact between the SIW and one of the waveguide parts. Furthermore, metamaterial-based gap waveguide technology provides a resonance-free packaging solution.
\end{abstract}

\section{INTRODUCTION}

There is a strong desire to increase the bandwidth and thereby the data rate of wireless communication systems. This has led the researchers and engineers to shift toward the millimeter-wave band or higher. Integrating active components such as an MMIC in a waveguide at millimeter-wave frequencies is often critical in terms of packaging as well as the transition between the chip's transmission lines and the waveguide. Transitions from microstrip lines to waveguides have gained a lot of interest. In the last decades, several papers have been presented in this direction. Some of these transitions are based on microstrip line to conventional ridge waveguides [1], [2]. These transitions perform well, however, such transitions need metal contacts between the microstrip line and the waveguide. Moreover, partially dielectric-filled waveguide cause manufacturing problems at higher frequencies. A microstrip to conventional waveguide transition at high frequencies is typically very complex because it requires an accurate alignment and good electrical joints when manufacturing and assembling split block sections of conventional waveguides. By introducing the gap waveguide technology [3], [4], critical electric contact and cavity-mode resonance can be avoided [5]. Recently, many transitions from microstrip line to gap waveguide components have been developed. A Ku-band microstrip line to ridge gap waveguide with $0.5 \mathrm{~dB}$ insertion loss has been designed [6]. This structure is complicated since the field needs to be coupled between the microstrip line to ridge gap waveguide through a slot. Furthermore, a back-short is needed which makes the structure bulky and also reduces the bandwidth. Another microstrip line to ridge gap waveguide transition operating at Ka-band is presented in [7]. This transition gives larger bandwidth than [6]. However, accurate alignment and electrical connection between the microstrip line and the ridge gap waveguide must be achieved. A contactless microstrip to ridge gap waveguide transition operating at $100 \mathrm{GHz}$ is presented in [8].However, an accurate alignment between the microstrip

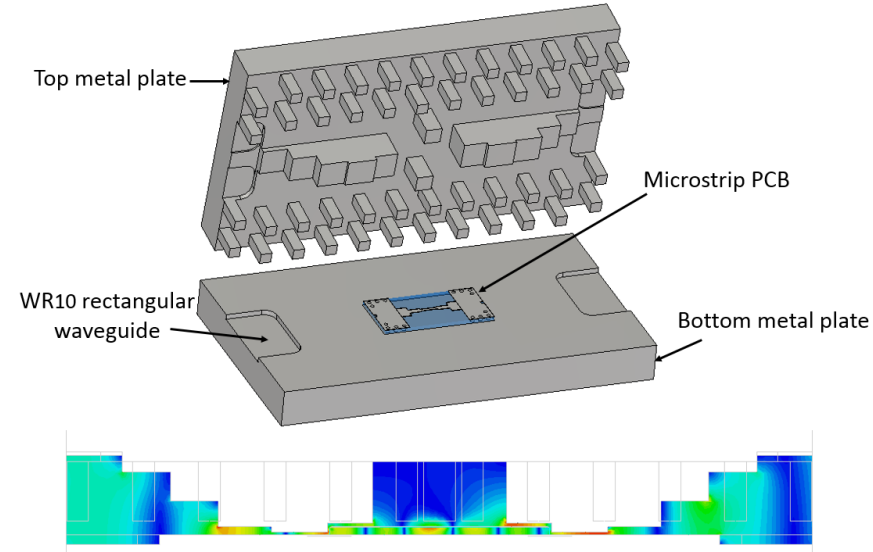

Fig. 1. The configuration of the proposed microstrip-to-groove gap waveguide transition and side view of the field guided through the structure

patch and the ridge section. Furthermore, the transition exhibits a relatively narrow bandwidth.

It is therefore the objective of this paper to provide a new compact and broadband contactless microstrip line to groove gap waveguide transition which is less susceptible to alignment problems and which particularly allows assembly in a fast and an easy manner, for example using a pick-and-place technique, as depicted in Fig. 1

\section{DESIGN OF THE PROPOSED TRANSITION}

The proposed back-to-back microstrip line to groove gap waveguide transition comprises a PCB of high permittivity substrate, attached to the bottom metal plate. The PCB comprises a transition of microstrip line to SIW and the gap waveguide structure comprises a transition of ridge gap to groove gap waveguide as shown in Fig. 2.

Both the SIW and microstrip PCB employ the same alumina substrate with dielectric constant of $\epsilon_{r}=9.9$, which is comparable to most of the MMICs used at millimeter-wave frequencies.

Electromagnetic coupling of the EM field between the SIW and the ridge gap waveguide is achieved by using a $\lambda_{g} / 4$ stub positioned at a slight distance above the PCB and also at a slight distance from the SIW edge, such that there is 


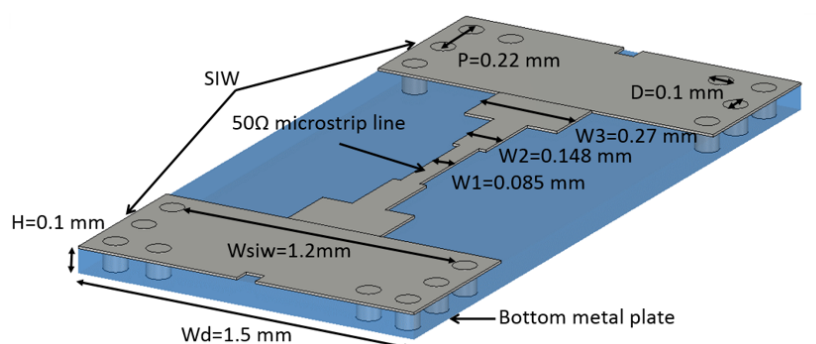

(a)

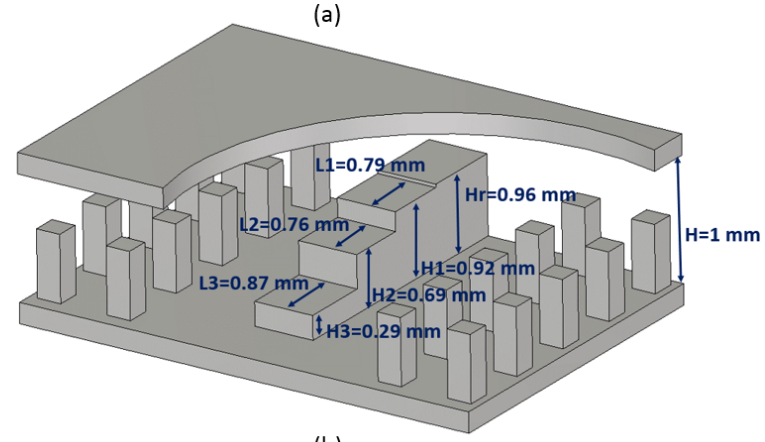

(b)

Fig. 2. (a) Back-to-back Microstrip to SIW transition, (b) Ridge gap to groove gap waveguide transition

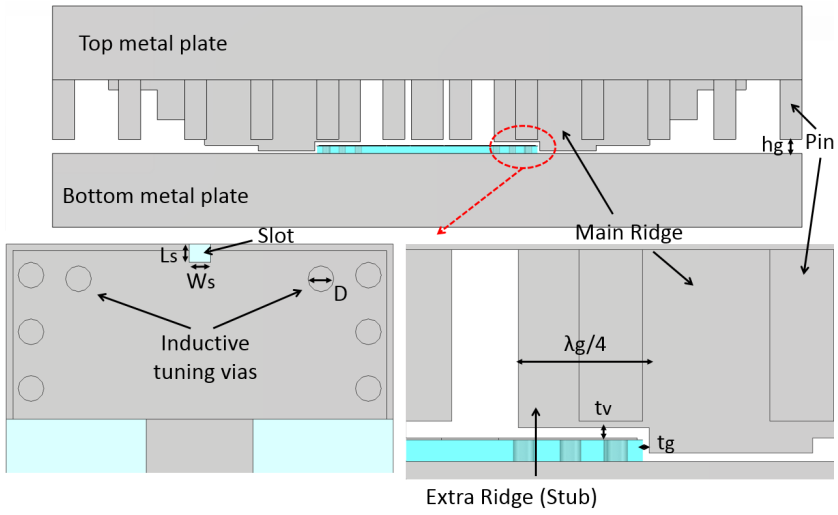

Fig. 3. Back-to-back transition, (zoomed) Slot size in the SIW ground plane, $W_{s}=0.083 \mathrm{~mm}, L_{s}=0.071 \mathrm{~mm}$, (zoomed) the air gaps in vertical, $t_{v}=0.048 \mathrm{~mm}$ and in horizontal, $t_{g}=0.03 \mathrm{~mm}$ and $h_{g}=0.19 \mathrm{~mm}$

an air gap between the $\lambda_{g} / 4$ open-ended stub and the SIW, effectively providing a short-circuit connection. Two inductive tuning vias and a U-shaped slot, as seen in Fig. 3, are used to compensate for the capacitance of the stub, thereby increasing the operational bandwidth. The commercial full-wave software CST Microwave Studio was used to design and simulate the transition. The simulation results of the back-to-back transition are shown in Fig. 4. The insertion loss and the return loss values are below $0.35 \mathrm{~dB}$ and larger than $21 \mathrm{~dB}$ over the entire $\mathrm{W}$-band, respectively (PEC materials).

\section{CONCLUSIONS}

A novel concept to realize a contactless microstrip line to groove gap waveguide transition through near-field electromagnetic coupling has been designed and simulated. The Quasi-TEM microstrip line mode is well-matched to the $T E_{10}$ mode of the SIW waveguide, which in turn is coupled to the ridge gap waveguide mode via a tuning stub partially

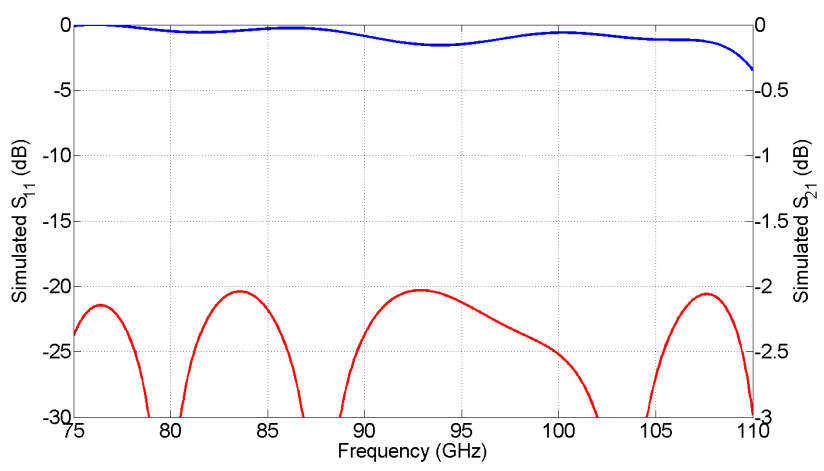

Fig. 4. Simulation result of proposed back-to-back transition

overlapping the PCB. Simulation results have shown an excellent performance; the simulated port reflection is smaller than $1 \%$ and the power transmission coefficient is larger than $99 \%$ over the entire W-band. The PCB part is directly packaged within the gap waveguide structure, so that there is no need for an additional packaging step. This is of significant importance when integrating MMICs operating at millimeterwave frequencies.

\section{ACKNOWLEDGMENT}

The work has been funded by a VR Young Researcher grant, the European Research Council (ERC) under the 7th framework programme ERC Grant Number 321125 and the Swedish Governmental Agency for Innovation Systems (VINNOVA) within the VINN Excellence Center Chase at Chalmers.

\section{REFERENCES}

[1] Y. Zhang, J. Ruiz-Cruz, K. Zaki, and A. Piloto, "A waveguide to microstrip inline transition with very simple modular assembly," IEEE Microw. Wireless Compon. Lett., vol. 20, no. 9, pp. 480-482, Sep. 2010.

[2] A. Rebollo, B. Larumbe-Gonzalo, R. Gonzalo, and I. Ederra, "Full wband microstrip-to-waveguide inline transition," in The $8^{r t} h$ European Conference on Antennas and Propagation (EuCAP 2014), pp. 25912593.

[3] P.-S. Kildal, E. Alfonso, A. Valero-Nogueira, and E. Rajo-Iglesias, "Local metamaterial-based waveguides in gaps between parallel metal plates," IEEE Antennas Wireless Propag. Lett., vol. 8, pp. 84-87, 2009.

[4] P.-S. Kildal, "Three metamaterial-based gap waveguides between parallel metal plates for mm/submm waves," in Proceedings of the $3^{r d}$ European Conference on Antennas and Propagation (EuCAP 2009), pp. 28-32.

[5] E. Rajo-Iglesias, A. Zaman, and P.-S. Kildal, "Parallel plate cavity mode suppression in microstrip circuit packages using a lid of nails," IEEE Microw. Wireless Compon. Lett., vol. 20, no. 1, pp. 31-33, Jan. 2010.

[6] B. Molaei and A. Khaleghi, "A novel wideband microstrip line to ridge gap waveguide transition using defected ground slot," IEEE Microw. Wireless Compon. Lett., vol. 25, no. 2, pp. 91-93, Feb. 2015.

[7] A. Zaman, T. Vukusic, M. Alexanderson, and P.-S. Kildal, "Design of a simple transition from microstrip to ridge gap waveguide suited for mmic and antenna integration," IEEE Antennas Wireless Propag. Lett., vol. 12, pp. 1558-1561, Dec. 2013.

[8] A. A. Brazlez, A. Zaman, and P.-S. Kildal, "Investigation of a microstripto-ridge gap waveguide transition by electromagnetic coupling," in AP-S International Symposium, IEEE Antennas and Propagation Society, Jul. 2012, pp. 1-2. 